AperTO - Archivio Istituzionale Open Access dell'Università di Torino

Can observational growth rate data favor the clustering dark energy models?

This is a pre print version of the following article:

Original Citation:

Availability:

This version is available http://hdl.handle.net/2318/1842129

since 2022-07-12T11:08:41Z

Published version:

DOI:10.1007/s10509-014-2185-3

Terms of use:

Open Access

Anyone can freely access the full text of works made available as "Open Access". Works made available under a Creative Commons license can be used according to the terms and conditions of said license. Use of all other works requires consent of the right holder (author or publisher) if not exempted from copyright protection by the applicable law. 


\title{
Can observational growth rate data favour the clustering dark energy models?
}

\author{
Ahmad Mehrabi ${ }^{1} \cdot$ Mohammad Malekjani $^{1}$ • \\ Francesco Pace ${ }^{2}$
}

\begin{abstract}
Under the commonly used assumption that clumped objects can be well described by a spherical top-hat matter density profile, we investigate the evolution of the cosmic growth index in clustering dark energy (CDE) scenarios on sub-horizon scales. We show that the evolution of the growth index $\gamma(z)$ strongly depends on the equation-of-state (EoS) parameter and on the clustering properties of the dark energy (DE) component. Performing a $\chi^{2}$ analysis, we show that CDE models have a better fit to observational growth rate data points with respect to the concordance $\Lambda \mathrm{CDM}$ model. We finally determine $\gamma(z)$ using an exponential parametrization and demonstrate that the growth index in CDE models presents large variations with cosmic redshift. In particular it is smaller (larger) than the theoretical value for the $\Lambda \mathrm{CDM}$ model, $\gamma_{\Lambda} \simeq 0.55$, in the recent past (at the present time).
\end{abstract}

\section{Introduction}

The current accelerated expansion of the universe is one of the biggest challenges in modern cosmologies. A wide range of astronomical data, including supernova type Ia (SNeIa) (Perlmutter et al. 1997, 1998, 1999; Riess et al. 2004, 2007), cosmic microwave background (CMB)

Ahmad Mehrabi

E-mail: mehrabi@basu.ac.ir

Mohammad Malekjani

E-mail: malekjani@basu.ac.ir

Francesco Pace

E-mail: francesco.pace@port.ac.uk

${ }^{1}$ Department of Physics, Faculty of Science, Bu-Ali Sina University, Hamedan 65178, Iran

${ }^{2}$ Institute of Cosmology and Gravitation, University of Portsmouth, Dennis Sciama Building, Portsmouth, PO1 3FX, U.K.
(Bennett et al. 2003; Spergel et al. 2003, 2007), large scale structures (LSS) (Hawkins et al. 2003; Tegmark et al. 2004; Cole et al. 2005) and baryon acoustic oscillations (BAO) (Eisenstein et al. 2005) confirm the current accelerated expansion of the universe. This is a surprising result, since in a universe dominated by matter, and therefore where gravity is attractive, we should expect the acceleration to slow down. Within the framework of General Relativity (GR), the accelerated expansion can be accommodated by the introduction of an unknown form of energy with sufficiently negative pressure, the so called dark energy (DE). Observational results of the Planck satellite (Planck Collaboration et al. 2013) indicate that $\mathrm{DE}$ is $\approx 68 \%$ of the total energy budget of the universe, while pressureless matter (baryons and cold dark matter) amount to $\approx 32 \%$. Hence, at the present time the dominant component is the $\mathrm{DE}$ with a negative equation of state $(\operatorname{EoS}) w_{\mathrm{de}}=p_{\mathrm{de}} / \rho_{\mathrm{de}}<-1 / 3$.

A very simple and important example of fluid with negative $\operatorname{EoS}$ is Einstein's cosmological constant, $\Lambda$, with $w_{\Lambda}=-1$ independent of time (Weinberg 1989; Sahni \& Starobinsky 2000; Peebles \& Ratra 2003). Although the cosmological constant is consistent with observational data, it suffers from the fine-tuning and the cosmic coincidence problems (Weinberg 1989; Sahni \& Starobinsky 2000; Peebles \& Ratra 2003). Alternatively, extensions and/or modifications of Einstein gravity theory, such as $f(R)$ and $f(T)$ models, can explain the present cosmic acceleration Amendola et al. 2007; Hu \& Sawicki 2007; Applebv \& Battve 2007; Starobinskv| 2007; Tsujikawa et al. 2008; Cognola et al. 2008; Linder 2009). In a general relativistic framework, DE models with equation of state $w_{\text {de }} \neq-1$ have been proposed, usually in the context of scalar fields such as quintessence models, in order to solve or alleviate the cosmological constant problems Peebles \& Ratra 2003). Moreover, phenomenological models with time evolving EoS parameter $w_{\text {de }}(z)$ have been suggested 
Linder 2003; Sahni et al. 2003; Alam et al. 2003; Lazkoz et al. 2005).

DE not only accelerates the expansion rate of the universe but also changes the growth rate of matter perturbations of cosmic structures. In fact, the growth of matter perturbation $\delta_{\mathrm{m}}$ of overdense regions is slowed down by the Hubble drag in an expanding universe (Peebles 1993). Hence, increasing the Hubble parameter in DE cosmologies causes an increasingly lower growth of structures. If $\mathrm{DE}$ is not the cosmological constant, we can assume fluctuations in both time and space (Pace et al. 2010), in a similar fashion to what happens for matter. These fluctuations will therefore have an impact on the growth of matter perturbation Abramo et al. 2009). This is due to the fact that DE is now not only important at the background level, but its fluctuations can enhance or dampen also fluctuations in the dark matter fluid.

The key parameter to describe perturbations for the DE fluid is the effective sound speed $c_{\text {eff }}^{2}=\delta p_{\text {de }} / \delta \rho_{\text {de }}$. In the context of canonical scalar fields, $c_{\text {eff }}^{2}=1$ (in units of the light speed, $c$ ), so that DE perturbations take place on scales equal to or larger than the horizon and are totally negligible on smaller scales $(\mathrm{Hu} 1998)$. However, for non-canonical scalar fields, like k-essence models, the effective sound speed is different from 1 , so that we can consider $c_{\text {eff }}^{2}<1$. In this case, DE perturbations can grow and be significant also on sub-horizon scales (Armendariz-Picon et al. 2001). In particular, when $c_{s}^{2} \ll 1$, perturbations can grow at the same rate as matter perturbations (Abramo et al. 2009). Hence, one can consider the impact of DE perturbations on the formation of cosmic structure on sub-horizon scales. In this work we focus on the effect of DE perturbations on the growth rate of matter in linear regime.

The growth of matter perturbation is described by the growth rate function $f=d \ln \delta_{\mathrm{m}} / d \ln a$, usually parametrized with the phenomenological functional form $f=\Omega_{\mathrm{m}}^{\gamma}$, where $\Omega_{\mathrm{m}}$ is the fractional energy density of matter in the universe and $\gamma$ is the socalled growth index (Peebles 1980, 1984; Lahav et al. 1991). The growth index for the concordance $\Lambda$ CDM model is approximately scale independent and constant with value $\gamma \simeq 0.55$ (Wang \& Steinhardt 1998; Linder 2005; Linder \& Cahn 2007). Modified gravity models have $0.40 \leq \gamma \leq 0.43$ at the present time $z=0$ (Tsujikawa et al. 2009; Gannouii et al. 2009). In the context of the Dvali-Gadabadze-Porrati (DGP) braneworld gravity model (Dvali et al. 2000), the growth index is $\gamma \simeq 11 / 16 \simeq 0.68$ (Linder \& Cahn 2007). Note, however, that this model has essentially been ruled out by observations, e.g. by the WiggleZ Dark Energy survey (Shi et al. 2012; Lombriser et al. 2013).
In the framework of General Relativity with dark energy characterised by a constant equation-of-state parameter, the growth index $\gamma$ is theoretically approximated by $\gamma \simeq \frac{3\left(w_{\mathrm{de}}-1\right)}{6 w_{\mathrm{d}}}$ (Silveira \& Waga 1994; Wang \& Steinhardt 1998; Linder 2004; Linder \& Cahn 2007; Nesseris \& Perivolaropoulos 2008; Lee \& Ng 2010) which explicitly reduces to the well known value $\gamma_{\Lambda} \simeq 6 / 11$ in the traditional $\Lambda \mathrm{CDM}$ cosmology with $w_{\Lambda}=-1$. Observationally, the growth rate $f(z)$ has been used to constrain DE models and the growth index $\gamma$ Nesseris \& Perivolaropoulos 2008; Cai et al. 2012). On the basis of observations, a wide range of values for $\gamma=(0.58-0.67)_{-0}^{+0.11+0.20}$ has been obtained (Nesseris \& Perivolaropoulos 2008: Guzzo et al. 2008; Dossett et al. 2010; Samushia et al. 2012; Hudson \& Turnbull 2012).

The weakness in using the observed values $f_{\text {obs }}(z)$ of the growth rate is due to the fact that it is model dependent, so that they can only be used to test the consistency of the $\Lambda \mathrm{CDM}$ model. Here, we rather prefer to use the quantity $f(z) \sigma_{8}(z)$, where $\sigma_{8}(z)$ is the time-dependant rms amplitude of the overdensity $\delta_{\mathrm{m}}$ at the comoving scale of $8 h^{-1}$ Mpc. The advantage is that this quantity is almost model independent and therefore suits better in constraining DE models (Song \& Percival 2009). Using the observational growth data $f(z) \sigma_{8}(z)$, we will constraint CDE models with equation of state $w_{\text {de }}$. As mentioned before, in CDE models the sound horizon of DE perturbations is well within the Hubble scale $H^{-1}$ and DE clusters on scales outside its sound horizon and smaller than the Hubble length (Appleby et al. 2013). In the same way, cold dark matter clumps on scales greater than its own Jeans scale. Instead for canonical DE models, the sound horizon is equal to or larger than the Hubble length and DE perturbations cannot grow on subHorizon scales. In this work we explore the signature of CDE models on the growth index of cosmic structures. We put constraints on the growth index $\gamma$ for a given CDE model and compare the results with the standard $\Lambda \mathrm{CDM}$ scenario. We will show that in CDE models, $\gamma$ may have substantial variations with cosmic redshift, so that the usual constant growth index parametrization is in general not suitable in these cases. Due to the inaccuracy of a constant $\gamma$ parametrization in $\mathrm{CDE}$, we use a general redshift dependent exponential parametrization and obtain the best fit values of the parameters.

The plan of the paper is as follows. In Sect. 2 we present the coupled differential equations governing the perturbations of matter and $\mathrm{DE}$ and argue about the growth index in such a system. The effect of DE perturbations on the growth index is discussed. In Sect. 3 we constrain the EoS parameter of DE for two different models, with constant and time-varying equation 
of state, by means of the $\chi^{2}$ analysis. We then evaluate $f(z) \sigma_{8}(z)$ and compare our theoretical prediction with observational data. Our results show that perturbations in the DE component give a better fit to observational data with respect to the $\Lambda \mathrm{CDM}$ model. We finally conclude in Sect. 4 .

\section{Growth index and dark energy perturbation}

In an Einstein-de Sitter (EdS) universe, in the linear perturbation theory for scales much smaller than the Hubble radius, the following differential equation describes the evolution of the perturbations of pressureless matter overdensities $\delta_{\mathrm{m}}=\delta \rho_{\mathrm{m}} / \rho_{\mathrm{m}}$ Linder \& Cahn 2007; Linder 2005; Huterer \& Linder 2007; Wang \& Steinhardt 1998; Nesseris \& Perivolaropoulos 2008; Chiba \& Takahash 2007):

$\ddot{\delta}_{\mathrm{m}}+2 H \dot{\delta}_{\mathrm{m}}-4 \pi G \rho_{\mathrm{m}} \delta_{\mathrm{m}}=0$.

It is well known that Eq. (1D) is still valid for DE cosmologies where its effects are only at the background level. In this case the DE fluid only modifies the Hubble function $\mathrm{H}$. This is not the case any more when DE perturbations kick in.

In the case of $\mathrm{CDE}$ models, we use the following set of linear coupled differential equations obtained in the framework of the spherical collapse model with the usual top-hat approximation by Abramo et al. (2009):

$$
\begin{aligned}
& \ddot{\delta}_{\mathrm{m}}+2 H \dot{\delta}_{\mathrm{m}}= \\
& \quad \frac{3 H^{2}}{2}\left[\Omega_{\mathrm{m}} \delta_{\mathrm{m}}+\Omega_{\mathrm{de}} \delta_{\mathrm{de}}\left(1+3 w_{\mathrm{de}}\right)\right], \\
& \ddot{\delta}_{\mathrm{de}}+\left(2 H-\frac{\dot{w}_{\mathrm{de}}}{1+w_{\mathrm{de}}}\right) \dot{\delta}_{\mathrm{de}}= \\
& \quad \frac{3}{2} H^{2}\left(1+w_{\mathrm{de}}\right)\left[\Omega_{\mathrm{m}} \delta_{\mathrm{m}}+\Omega_{\mathrm{de}} \delta_{\mathrm{de}}\left(1+3 w_{\mathrm{de}}\right)\right],
\end{aligned}
$$

where overdot denotes the derivative with respect to cosmic time $t, \delta_{\mathrm{de}}$ and $w_{\mathrm{de}}$ indicate the perturbations and the EoS parameter of DE, respectively. Under the Top-Hat approximation, $\delta_{\mathrm{m}}$ and $\delta_{\mathrm{de}}$ are uniform inside the perturbed region and therefore are a function of cosmic time only. It should be emphasized that perturbation theory based on the Top-Hat approximation is fully consistent with GR and Pseudo-Newtonian cosmology in the linear regime (Abramo et al. 2009). Indeed, Abramo et al. (2009) showed that the growing modes of perturbations in GR and in the PseudoNewtonian formalism are the same and differences appear only in the decaying mode. Using the relation $\frac{d}{d t}=a H \frac{d}{d a}$, Eqs. (2 \& 3) can be written as

$$
\begin{aligned}
& a^{2} \delta_{\mathrm{m}}^{\prime \prime}+\frac{3}{2} a\left(1-\Omega_{\mathrm{de}} w_{\mathrm{de}}\right) \delta_{\mathrm{m}}^{\prime}= \\
& \frac{3}{2}\left[\Omega_{\mathrm{m}} \delta_{\mathrm{m}}+\Omega_{\mathrm{de}} \delta_{\mathrm{de}}\left(1+3 w_{\mathrm{de}}\right)\right] \\
& a^{2} \delta_{\mathrm{de}}^{\prime \prime}+\frac{3}{2} a\left(1-\Omega_{\mathrm{de}} w_{\mathrm{de}}-\frac{a w_{\mathrm{de}}^{\prime}}{1+w_{\mathrm{de}}}\right) \delta_{\mathrm{de}}^{\prime}= \\
& \quad \frac{3}{2}\left(1+w_{\mathrm{de}}\right)\left[\Omega_{\mathrm{m}} \delta_{\mathrm{m}}+\Omega_{\mathrm{de}} \delta_{\mathrm{de}}\left(1+3 w_{\mathrm{de}}\right)\right],
\end{aligned}
$$

where $a$ is the scale factor and primes denote the derivative with respect to $a$. To derive Eqs. (2) and (3), the EoS parameter of DE, $w_{\text {de }}$ is assumed to be the same inside and outside the perturbed region. We therefore note that it is possible to derive this set of equations simdt by modifying the source term in Poisson equation qq. 4) in Pace et al. (2010). To evaluate the impact of DE perturbations as well as the EoS parameter $w_{\text {de }}$ on the growth rate of structures in CDE models, we solve Eqs. (4)and 5) numerically and obtain $\delta_{\mathrm{m}}(z)$ and $\delta_{\mathrm{de}}(z)$ where $z$ is the cosmic redshift related to the scale factor by $z=1 / a-1$.

For simplicity, in this section we choose a constant EoS parameter for the dark energy models investigated. We analyse two different regimes: the phantom one $\left(w_{\mathrm{de}}=-1.2\right)$ and the quintessence one $\left(w_{\mathrm{de}}=-0.8\right)$. A more general dark energy model with time varying EoS parameter $w_{\mathrm{de}}(z)$ will be discussed in next section. We chose the following adiabatic initial conditions at early times $\left(z_{\mathrm{i}}=1000\right)$ : $\delta_{\mathrm{m}}\left(z_{\mathrm{i}}\right) \simeq 1.4 \times 10^{-4}, \delta_{m}^{\prime}\left(z_{\mathrm{i}}\right) \simeq-\delta_{\mathrm{m}}\left(z_{\mathrm{i}}\right) /\left(1+z_{\mathrm{i}}\right)$ and $\delta_{\mathrm{de}}\left(z_{\mathrm{i}}\right)=\frac{\left(1+w_{\mathrm{de}}\right)}{1+3 w_{\mathrm{e}}} \delta_{\mathrm{m}}\left(z_{\mathrm{i}}\right), \delta_{\mathrm{de}}^{\prime}\left(z_{\mathrm{i}}\right) \simeq-\delta_{\mathrm{de}}\left(z_{\mathrm{i}}\right) /\left(1+z_{\mathrm{i}}\right)$ (Spergel et al. 2007). To solve the equations, we set the present values for the matter and the DE density parameters as $\Omega_{\mathrm{m}, 0}=0.3175$ and $\Omega_{\mathrm{de} .0}=0.6825$ provided by the WMAP experiments Komatsu et al. 2011). By calculating $\delta_{\mathrm{m}}(z)$ and using the definition $f(z)=d \ln \delta_{\mathrm{m}}(z) / d \ln a$ together with the parametrization $f(z)=\Omega_{\mathrm{m}}(z)^{\gamma(z)}$, we obtain the growth index $\gamma(z)$ from the coupled Eqs. (4) \& 5). In Fig. (11) we show the evolution of $\gamma(z)$ as a function of redshift $z$ for both phantom $w_{\mathrm{de}}=-1.2$ and non-phantom $w_{\mathrm{de}}=-0.8$ EoS parameters. The concordance $\Lambda \mathrm{CDM}$ cosmology is approximately constant, $\gamma \simeq 0.55$ (dashed green line) as expected. Analogously to the $\Lambda \mathrm{CDM}$ model, the growth index in non-clustering dark energy (Non-CDE) models is largely redshift-independent, both for phantom (blue dotted line) and quintessence (black shortdashed line) regimes. We also see that differences from the $\Lambda \mathrm{CDM}$ model are negligible in Non-CDE models. However, in CDE models, where $\delta_{\mathrm{de}}(z) \neq 0$ and non-negligible, the growth index changes with redshift and can become as high as $\gamma(z=0) \simeq 0.71$ for the 
quintessence case (violet solid curve) and as low as $\gamma(z=0) \simeq 0.38$ in the phantom case (dot-dashed red curve). It is also possible to appreciate the large deviation of $\gamma(z)$ in CDE models from the concordance $\Lambda \mathrm{CDM}$ value 0.55 as well. Here we conclude that the impact of DE perturbations on the growth rate of structures is comparable to the influence of the EoS parameter. Moreover, $\gamma(z)$ is larger (smaller) than $\Lambda \mathrm{CDM}$ value 0.55 in the case of quintessence (phantom) EoS parameter.

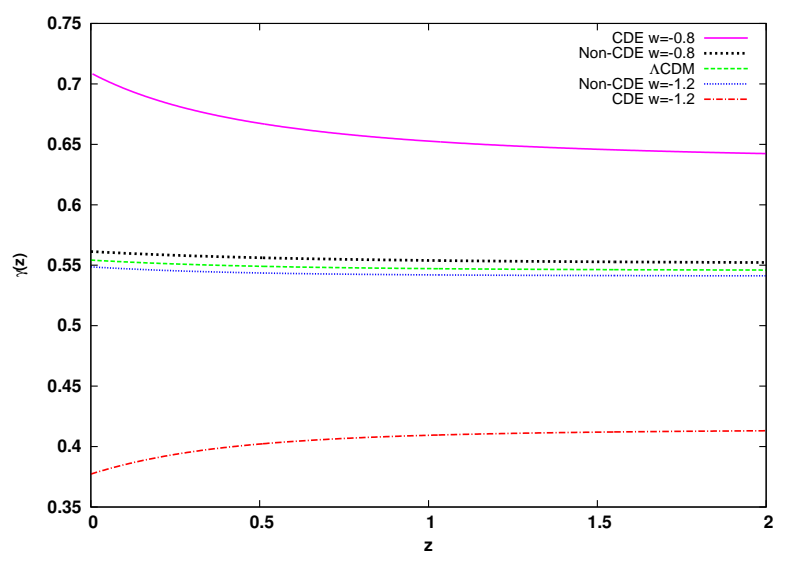

Fig. 1 The variation of the growth index $\gamma(z)$ as a function of the cosmic redshift $z$ for different CDE and Non-CDE models. The solid violet (black short-dashed) curve represents the clustering (non-clustering) quintessence model, the dashed green curve the reference $\Lambda$ CDM model, while the blue dotted (red dot-dashed) curve stands for the clustering (non-clustering) phantom dark energy model.

\section{Observational constraint on the growth index in CDE models}

In this section we use the current observational growth data reported in table (1) to find the best fit value for the parameters of the CDE models here studied. We first consider the CDE model with constant EoS parameter, namely the wCDE model. We then assume a time varying EoS parameter using the Chevallier-PolarskiLinder (CPL) parametrization Chevallier \& Polarski 2001; Linder 2003)

$w_{\mathrm{de}}(z)=w_{0}+w_{1}\left(\frac{z}{1+z}\right)$

the so-called $\mathrm{w}(\mathrm{t}) \mathrm{CDE}$ model. In the ${ }^{\mathrm{C} C D E}$ model, there is one free parameter (the equation of state $w_{\mathrm{de}}$ ) while for the $\mathrm{w}(\mathrm{t}) \mathrm{CDE}$ model we have two free parameters $\left(w_{0}, w_{1}\right)$. Considering these two models, we solve the system of Eqs. (4) \& 5 numerically to obtain the theoretical value for growth factor $f(z)_{\text {the, }}$ where the subscript "the" indicates the theoretical value.

We also calculate $\sigma_{8}(z)$ the as

$$
\begin{aligned}
\sigma_{8}(z=0) & =\frac{\delta_{\mathrm{m}}(z=0)}{\delta_{\mathrm{m}, \Lambda}(z=0)} \sigma_{8, \Lambda}(z=0), \\
\sigma_{8}(z) & =D_{+}(z) \sigma_{8}(z=0),
\end{aligned}
$$

where $D_{+}(z)=\delta_{\mathrm{m}}(z) / \delta_{\mathrm{m}}(z=0)$ is the growth factor. We assume $\sigma_{8, \Lambda}(z=0)=0.811$, in agreement with the WMAP-7 results (Komatsu et al. 2011).

We also calculate the least square parameter $\chi^{2}$ according to the definition

$\chi^{2}=\sum_{i} \frac{\left(\left[f(z) \sigma_{8}\left(z_{i}\right)\right]_{\mathrm{obs}}-\left[f(z) \sigma_{8}\left(z_{i}\right)\right]_{\mathrm{the}}\right)^{2}}{\sigma_{i}^{2}}$

where the subscript "obs" stands for the observational value and $\sigma_{i}$ is the uncertainty of the observational data. Currently there are only 10 data points for $f(z) \sigma_{8}$ in the redshift range $0.067 \leq z \leq 0.80$ as shown in table (1).

In Fig. (2) we show the evolution of $\chi^{2}(w)$ as a function of the dark energy equation of state $w_{\text {de }}$ for the wCDE models. Here we set the best value of $\chi^{2}$ to zero and show the $1-\sigma$ and $2-\sigma$ confidence levels by a cyan and an orange line, respectively. For the wCDE model we obtain the following best fit value for the EoS parameter: $w_{\mathrm{de}}=-0.764_{-0.072-0.136}^{+0.073+0.169}$ with a $\chi_{\text {best }}^{2}=6.39$, while for $\mathrm{w}(\mathrm{t}) \mathrm{CDE}$ model we obtain $w_{0}=-0.439_{-0.132-0.192}^{+0.078+0.109}, w_{1}=0.375_{-0.275-0.136}^{+0.175+0.239}$ with a $\chi_{\text {best }}^{2}=4.05$. In the case of the concordance $\Lambda \mathrm{CDM}$ model we find $\chi_{\text {best }}^{2}=18.58$ and for comparison we obtain a $\chi_{\text {best }}^{2}=147.09$ for the EdS model. The results of the $\chi^{2}$ analysis for the $\mathrm{w}(\mathrm{t}) \mathrm{CDE}$ model is presented in Fig. (3). As mentioned before, in this case there are two free parameters $w_{0}$ and $w_{1}$ to be constrained. The star symbol shows the location of the best fit value and the inner and outer contours represent the $1-\sigma$ and $2-\sigma$ confidence levels, respectively. In Fig. (4) we instead present results for the quantity $f(z) \sigma_{8}(z)$ for both theoretical models (lines) and observational data (points with error bars).

In order to select the best model among the ones here studied, we should compute the reduced chi-squared parameter $\chi_{\text {red }}^{2}=\chi_{\text {best }}^{2} / \nu$, where $\nu$ represents the number of degrees of freedom and it is given by $\nu=N-n-1$, where $N$ is the number of data points and $n$ is the number of fitted parameters. The deviation from $\chi_{\text {red }}^{2}=1$ measures how good the model is. For all the models $N=10$. For the wCDE model $n=4\left(\Omega_{\mathrm{m}, 0}, \Omega_{\mathrm{de}, 0}\right.$, $\left.\sigma_{8}, w_{\text {de }}\right)$. In the case of $\mathrm{w}(\mathrm{t}) \mathrm{CDE}$ models, $n=5$ $\left(\Omega_{\mathrm{m}, 0}, \Omega_{\mathrm{de}, 0}, \sigma_{8}, w_{0}, w_{1}\right)$. For the concordance $\Lambda \mathrm{CDM}$ 


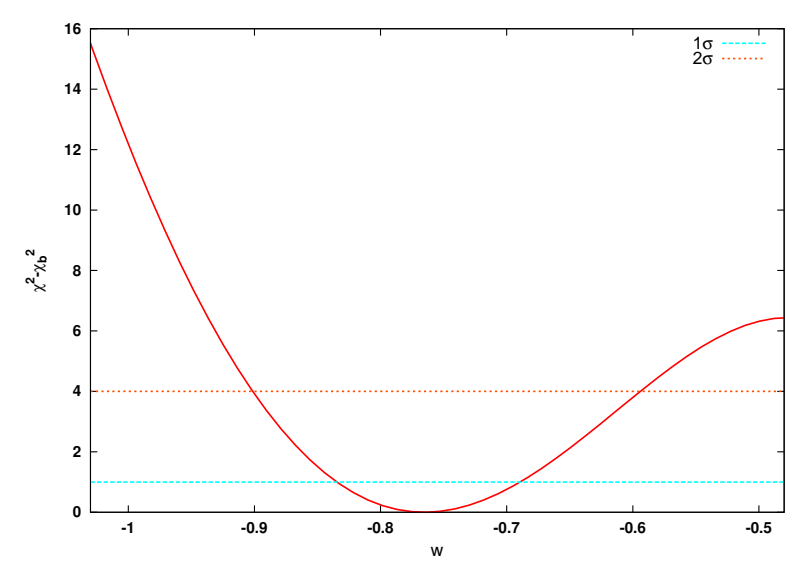

Fig. 2 Variation of $\chi^{2}-\chi_{\text {best }}^{2}$ as a function of $w$ for the wCDE models. The two horizontal lines show the $1-\sigma$ and the $2-\sigma$ confidence levels.

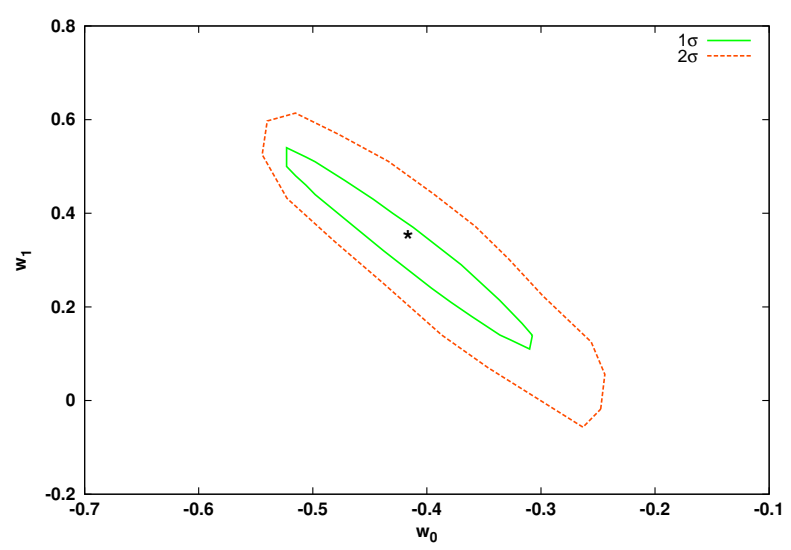

Fig. 3 Best parameter values and $1-\sigma$ and $2-\sigma$ confidence levels for the $\mathrm{w}(\mathrm{t}) \mathrm{CDM}$ model. The location of the minimum $\chi^{2}$ is shown a star.

model $n=3\left(\Omega_{\mathrm{m}, 0}, \Omega_{\mathrm{de}, 0}, \sigma_{8}\right)$ and for the EdS model $n=1\left(\sigma_{8}\right)$. We find the following values for the reduced chi-squared $\chi_{\nu}^{2}=6.39 / 5=0.79$ for the ${ }^{\mathrm{wCDE}}$ models, $\nu=4.05 / 4=1.01$ for the $\mathrm{w}(\mathrm{t}) \mathrm{CDE}$ models, $\nu=18.58 / 6=3.09$ for the concordance $\Lambda \mathrm{CDM}$ and $\nu=147.09 / 8=18.38$ for EdS scenarios. We can explicitly see that the CDE models fit better the data points than the standard $\Lambda \mathrm{CDM}$ and EdS scenarios. Moreover, the $\mathrm{w}(\mathrm{t}) \mathrm{CDE}$ model is the best model with 0.01 deviation from $\chi_{\text {red }}^{2}=1$.

We can now evaluate the growth index $\gamma(z)=\frac{\ln f(z)}{\ln \Omega_{\mathrm{m}}}$ on the basis of best fit value for $w_{\mathrm{de}}$ for both wCDE and $\mathrm{w}(\mathrm{t}) \mathrm{CDE}$ models. Results are shown in Fig. (5). The $\Lambda \mathrm{CDM}$ model is shown by the green dashed line and as expected is practically a constant. The pink dotted curve represents the wCDE model $(0.66 \leq \gamma(z) \leq 0.73$ in the redshift interval $0 \leq z \leq 2)$ and is $32 \%$ bigger than the present value of the $\Lambda \mathrm{CDM}$ model $(\gamma=0.55)$.

\begin{tabular}{|c|c|c|}
\hline $\bar{z}$ & $f(z) \sigma_{8}(z)$ & Survey and Refs \\
\hline 0.067 & $0.42 \pm 0.06$ & 6dFGRS (Beutler et al. 2012$)$ \\
\hline 0.17 & $0.51 \pm 0.06$ & (Percival et al. \\
\hline 0.22 & $0.42 \pm 0.07$ & WiggleZ (Blake et al. 2011) \\
\hline 0.25 & $0.39 \pm 0.05$ & $\begin{array}{l}\text { SDSS-LRG (Samushia et al. } \\
\text { 2012) }\end{array}$ \\
\hline 0.37 & $0.43 \pm 0.04$ & $\begin{array}{l}\text { SDSS-LRG Samushia et al. } \\
\text { 2012) }\end{array}$ \\
\hline 0.41 & $0.45 \pm 0.04$ & WiggleZ (Blake et al. 2011) \\
\hline 0.57 & $0.43 \pm 0.03$ & $\begin{array}{l}\text { BOSS-CMASS (Reid et al. } \\
2012)\end{array}$ \\
\hline 0.60 & $0.43 \pm 0.04$ & WiggleZ (Blake et al. 2011) \\
\hline 0.78 & $0.38 \pm 0.04$ & WiggleZ (Blake et al.|2011) \\
\hline 0.80 & $0.47 \pm 0.08$ & $\begin{array}{l}\text { VIPERS (de la Torre et al. } \\
2013)\end{array}$ \\
\hline
\end{tabular}

Table 1 The $f(z) \sigma_{8}(z)$ data points including their reference and survey.

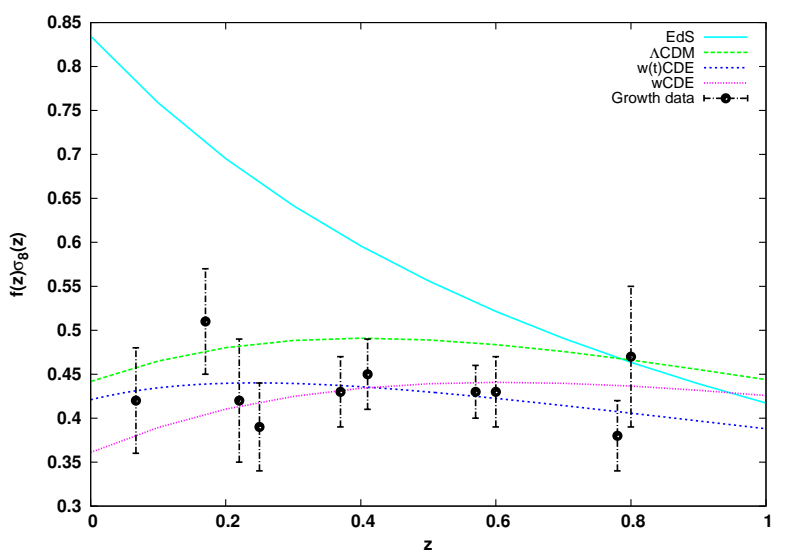

Fig. 4 The observed and the theoretical evolution of $f(z) \sigma_{8}$. The solid cyan curve stands for the Einstein-de Sitter (EdS) model, the green-dashed one indicates the $\Lambda$ CDM model, the pink and blue-dotted lines represent the wCDE and the $\mathrm{w}(\mathrm{t}) \mathrm{CDE}$ models, respectively. Circles with errorbars are the observed data points from table (1).

In the case of the $\mathrm{w}(\mathrm{t}) \mathrm{CDE}$ model, $\gamma(z)$ is $0.33 \leq \gamma(z) \leq$ 0.59 which is $9 \%$ bigger than the present value of the $\Lambda \mathrm{CDM}$ model (blue short-dashed curve). In this case $\gamma(z)$ crosses the constant $\Lambda \mathrm{CDM}$ line at $z \simeq 0.15$ representing a smaller growth index for $z>0.15$ and a bigger one for $z<0.15$ compared to the $\Lambda \mathrm{CDM}$ model. It is important to note that, unlike $\Lambda \mathrm{CDM}$ cosmologies, in CDE models the growth index $\gamma$ has a wide range of values, in particular in the case of $\mathrm{w}(\mathrm{t}) \mathrm{CDE}$ models. It should also be noted that for CDE models, due to the large variation of $\gamma(z)$ with respect to the redshift $z$, the constant $\gamma_{0}$ parametrization is not accurate enough. Hence, it is worth using a parametrized function of $\gamma(z)$ 
in order to discuss the best fit value of the growth index in CDE models. Using the fact that the growth index should be nearly constant at early times and change at late times, we apply the following general exponential parametrization (Dossett et al. 2010):

$\gamma(z)=\gamma_{\infty}+\gamma_{\mathrm{b}} e^{-z / z_{\mathrm{t}}}$

where $z_{\mathrm{t}}$ represents the transition redshift from an almost constant $\gamma_{\infty}$ at early times to a varying growth index $\gamma(z)$ at late times. In the case of the concordance $\Lambda$ CDM cosmology, the exponential parametrization yields the best fit parameters $\gamma_{\infty}=0.5457, \gamma_{\mathrm{b}}=$ 0.0103 and $z_{\mathrm{t}}=0.61$ (Dossett et al. 2010). In table 2. we show the best fit parameters of $\gamma_{\infty}, \gamma_{\mathrm{b}}$ and $z_{\mathrm{t}}$ with the corresponding error-bars for the ${ }_{\mathrm{wCDE}}$ and $\mathrm{w}(\mathrm{t}) \mathrm{CDE}$ models. A large value of $\gamma_{\mathrm{b}}$ represents a strong variation of $\gamma(z)$ with redshift for $\mathrm{w}(\mathrm{t}) \mathrm{CDE}$ model.

\begin{tabular}{ccl}
\hline Model & wCDM & $\mathrm{w}(\mathrm{t}) \mathrm{CDM}$ \\
\hline$\gamma_{\infty}$ & $0.648984_{-0.00006}^{+0.00006}$ & $0.253179_{-0.0004126}^{+0.0004126}$ \\
$\gamma_{\mathrm{b}}$ & $0.0750995_{-0.00021}^{+0.00021}$ & $0.318083_{-0.00055}^{+0.00055}$ \\
$z_{\mathrm{t}}$ & $0.698723_{-0.003559}^{+0.003559}$ & $1.39674_{-0.006793}^{+0.006793}$ \\
\hline
\end{tabular}

Table 2 The best fit parameters for $\gamma(z)$ on the basis of the parametrization of Eq. (10).

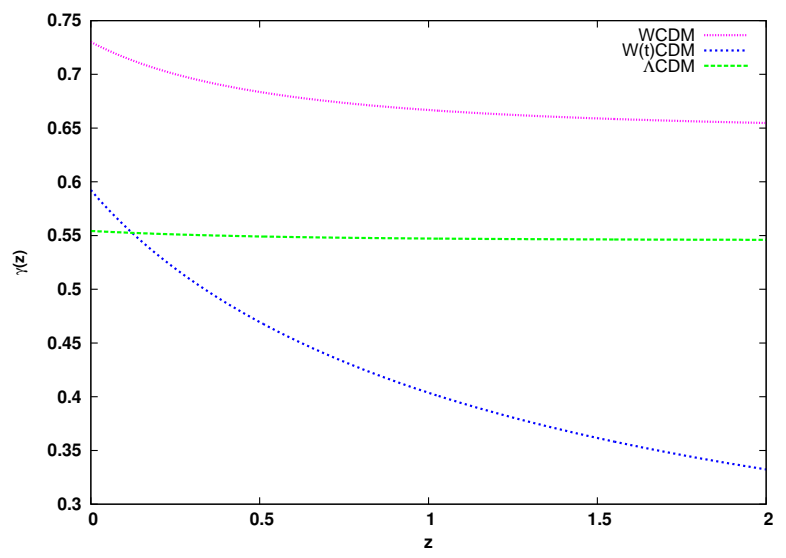

Fig. 5 Growth index for different cosmological models on the basis of best fit values of the EoS parameter.

\section{Conclusions}

In this work we studied the evolution of density perturbations of pressureless dark matter and dark energy on sub-horizon scales by solving the linear equations of structure formation with a density profile assumed to be described by a Top-Hat profile. We showed that the matter growth index $\gamma$ is strongly affected by perturbations of the DE component, while for smooth DE models it is almost constant with cosmic time. It has been shown that for CDE models, the growth index $\gamma$ can be larger (for quintessence EoS $w_{\mathrm{de}}>-1$ ) or smaller (for the phantom $\operatorname{EoS} w_{\mathrm{de}}<-1$ ) than the concordance $\Lambda \mathrm{CDM}$ predicted value $\gamma_{\Lambda} \simeq 0.55$. We performed a reduced $\chi^{2}$ minimization analysis between the observations and the theoretical expectations of $f(z) \sigma_{8}(z)$ and found that for CDE with constant EoS parameter, i.e., wCDE model: $w_{\mathrm{de}}=-0.764_{-0.072-0.136}^{+0.073+0.169}$ with a reduced $\chi_{\text {red }}^{2}=0.79$, while for $\mathrm{w}(\mathrm{t}) \mathrm{CDE}$ model with a CPL parametrization for $w_{\mathrm{de}}(z)$ Chevallier \& Polarski 2001; Linder 2003), we found: $\left(w_{0}=-0.439_{-0.132-0.192}^{+0.078+0.109}, w_{1}=\right.$ $\left.0.375_{-0.275-0.136}^{+0.175+0.239}\right)$ with a $\chi_{\text {red }}^{2}=1.01$. For comparison, the concordance $\Lambda$ CDM model has $\chi_{\text {red }}^{2}=3.09$. Hence the $\mathrm{w}(\mathrm{t}) \mathrm{CDE}$ model fits better to the observational growth data with respect to other models.

We also discussed the growth index $\gamma$ in the framework of clustering dark energy models and we found a strong variation with respect to redshift, particularly in the case of the $\mathrm{w}(\mathrm{t}) \mathrm{CDE}$ model. We showed that for the best fit value of $w_{\text {de }}$, the $\mathrm{w}(\mathrm{t}) \mathrm{CDE}$ model crosses the $\Lambda \mathrm{CDM}$ solution at $z \simeq 0.15$, with lower values for $z>0.15$ and higher values for $z<0.15$ when compared to the $\Lambda$ CDM result. Finally, we applied the general exponential parametrization for $\gamma(z)$ in Eq. (10) and predicted that for the wCDE model analysed $\gamma_{\infty}>\gamma_{\infty}^{\Lambda C D M}$, while for the w(t)CDE model $\gamma_{\infty}<\gamma_{\infty}^{\Lambda C D M}$ (see table 2). Hence the growth index at early times is larger for wCDE and smaller for $\mathrm{w}(\mathrm{t}) \mathrm{CDE}$ models compare with the $\Lambda$ CDM model. The large value of $\gamma_{\mathrm{b}}$ in table 2 indicates a strong variation of the growth index in $\mathrm{w}(\mathrm{t}) \mathrm{CDE}$ cosmologies.

\section{Acknowledgements}

F. Pace is supported by STFC grant ST/H002774/1. We would like to thank anonymous referee for giving constructive comments and reading the manuscript. 


\section{References}

Abramo, L.R., Batista, R.C., Liberato, L., Rosenfeld, R.: Phys. Rev. D 79(2), 023516 (2009)

Alam, U., Sahni, V., Deep Saini, T., Starobinsky, A.A.: Mon. Not. R. Astron. Soc. 344, 1057 (2003)

Amendola, L., Gannouji, R., Polarski, D., Tsujikawa, S.: Phys. Rev. D 75(8), 083504 (2007)

Appleby, S., Battye, R.: Physics Letters B 654, 7 (2007)

Appleby, S.A., Linder, E.V., Weller, J.: Phys. Rev. D 88(4), 043526 (2013)

Armendariz-Picon, C., Mukhanov, V., Steinhardt, P.J.: Phys. Rev. D 63(10), 103510 (2001)

Bennett, C.L., Halpern, M., Hinshaw, G., Jarosik, N., Kogut, A., Limon, M., Meyer, S.S., et al.: Astrophys. J. Suppl. Ser. 148, 1 (2003)

Beutler, F., Blake, C., Colless, M., Jones, D.H., StaveleySmith, L., Poole, G.B., Campbell, L., et al.: Mon. Not. R. Astron. Soc. 423, 3430 (2012)

Blake, C., Brough, S., Colless, M., Contreras, C., Couch, W., Croom, S., Davis, T., Drinkwater, M.J., Forster, K., et al.: Mon. Not. R. Astron. Soc. 415, 2876 (2011)

Cai, R.-G., Tuo, Z.-L., Wu, Y.-B., Zhao, Y.-Y.: Phys. Rev. D 86(2), 023511 (2012)

Chevallier, M., Polarski, D.: International Journal of Modern Physics D 10, 213 (2001)

Chiba, T., Takahashi, R.: Phys. Rev. D 75(10), 101301 (2007)

Cognola, G., Elizalde, E., Nojiri, S., Odintsov, S.D., Sebastiani, L., Zerbini, S.: Phys. Rev. D 77(4), 046009 (2008)

Cole, S., Percival, W.J., Peacock, J.A., Norberg, P., Baugh, C.M., Frenk, C.S., Baldry, I., Bland-Hawthorn, J., Bridges, T., et al.: Mon. Not. R. Astron. Soc. 362, 505 (2005)

de la Torre, S., Guzzo, L., Peacock, J.A., Branchini, E., Iovino, A., Granett, B.R., Abbas, U., Adami, C. Arnouts, S., et al.: Astron. Astrophys. 557, 54 (2013)

Dossett, J., Ishak, M., Moldenhauer, J., Gong, Y., Wang, A.: J. Cosmol. Astropart. Phys. 4, 22 (2010)

Dvali, G., Gabadadze, G., Porrati, M.: Physics Letters B 485, 208 (2000)

Eisenstein, D.J., Zehavi, I., Hogg, D.W., Scoccimarro, R., Blanton, M.R., Nichol, R.C., Scranton, R., Seo, H.-J., Tegmark, M., Zheng, Z., et al.: Astrophys. J. 633, 560 (2005)

Gannouji, R., Moraes, B., Polarski, D.: J. Cosmol. Astropart. Phys. 2, 34 (2009)

Guzzo, L., Pierleoni, M., Meneux, B., Branchini, E., Le Fèvre, O., Marinoni, C., Garilli, B., Blaizot, J., De Lucia, G., et al.: Nature 451, 541 (2008)

Hawkins, E., Maddox, S., Cole, S., Lahav, O., Madgwick, D.S., Norberg, P., Peacock, J.A., Baldry, I.K., Baugh, C.M., Bland-Hawthorn, J., et al.: Mon. Not. R. Astron.Soc. 346, 78 (2003)

Hu, W.: Astrophys. J. 506, 485 (1998)

Hu, W., Sawicki, I. : Phys. Rev. D 76(6), 064004 (2007)

Hudson, M.J., Turnbull, S.J.: Astrophys. J. Lett. 751, 30 (2012)

Huterer, D., Linder, E.V.: Phys. Rev. D 75(2), 023519 (2007)
Komatsu, E., Smith, K.M., Dunkley, J., et al.: Astrophys. J. Suppl. Ser. 192, 18 (2011)

Lahav, O., Lilje, P.B., Primack, J.R., Rees, M.J.: Mon. Not. R. Astron. Soc. 251, 128 (1991)

Lazkoz, R., Nesseris, S., Perivolaropoulos, L.: J. Cosmol. Astropart. Phys. 11, 10 (2005)

Lee, S., Ng, K.-W. : Physics Letters B 688, 1 (2010)

Linder, E.V.: Physical Review Letters 90(9), 091301 (2003)

Linder, E.V.: Phys. Rev. D 70(2), 023511 (2004)

Linder, E.V.: Phys. Rev. D 72(4), 043529 (2005)

Linder, E.V.: Phys. Rev. D 80(12), 123528 (2009)

Linder, E.V., Cahn, R.N.: Astroparticle Physics 28, 481 (2007)

Lombriser, L., Yoo, J., Koyama, K.: Phys. Rev. D 87(10), 104019 (2013)

Nesseris, S., Perivolaropoulos, L.: Phys. Rev. D 77(2), 023504 (2008)

Pace, F., Waizmann, J.-C., Bartelmann, M.: Mon. Not. R. Astron. Soc. 406, 1865 (2010)

Peebles, P.J., Ratra, B.: Reviews of Modern Physics 75, 559 (2003)

Peebles, P.J.E.: The Large-scale Structure of the Universe, (1980)

Peebles, P.J.E.: Astrophys. J. 284, 439 (1984)

Peebles, P.J.E.: Principles of Physical Cosmology, (1993)

Percival, W.J., Burkey, D., Heavens, A., Taylor, A., Cole, S., Peacock, J.A., Baugh, C.M., et al.: Mon. Not. R. Astron. Soc. 353, 1201 (2004)

Perlmutter, S., Gabi, S., Goldhaber, G., Goobar, A., Groom, D.E., Hook, I.M., Kim, A.G., et al.: Astrophys. J. 483, 565 (1997) astro-ph/9608192

Perlmutter, S., Aldering, G., della Valle, M., Deustua, S., Ellis, R.S., Fabbro, S., Fruchter, A., Goldhaber, G., et al.P: Nature 391, 51 (1998)

Perlmutter, S., Aldering, G., Goldhaber, G., et al.: Astrophys. J. 517, 565 (1999)

Planck Collaboration, Ade, P.A.R., Aghanim, N., ArmitageCaplan, C., Arnaud, M., Ashdown, M., Atrio-Barandela, F., Aumont, J., Baccigalupi, C., Banday, A.J., et al.: ArXiv e-prints, 1303.5076 (2013) 1303.5076

Reid, B.A., Samushia, L., White, M., Percival, W.J., Manera, M., Padmanabhan, N., Ross, A.J., et al.: Mon. Not. R. Astron. Soc. 426, 2719 (2012)

Riess, A.G., Strolger, L.-G., Tonry, J., Casertano, S., Ferguson, H.C., et al.: Astrophys. J. 607, 665 (2004)

Riess, A.G., Strolger, L.-G., Casertano, S., Ferguson, H.C., Mobasher, B., Gold, B., Challis, P.J., et al.: Astrophys. J. 659, 98 (2007)

Sahni, V., Starobinsky, A.: International Journal of Modern Physics D 9, 373 (2000)

Sahni, V., Saini, T.D., Starobinsky, A.A., Alam, U.: Soviet Journal of Experimental and Theoretical Physics Letters 77, 201 (2003)

Samushia, L., Percival, W.J., Raccanelli, A.: Mon. Not. R. Astron. Soc. 420, 2102 (2012)

Shi, K., Huang, Y.F., Lu, T.: Mon. Not. R. Astron. Soc. 426, 2452 (2012)

Silveira, V., Waga, I.: Phys. Rev. D 50, 4890 (1994)

Song, Y.-S., Percival, W.J.: J. Cosmol. Astropart. Phys. 10, 4 (2009) 
Spergel, D.N., Verde, L., Peiris, H.V., Komatsu, E., Nolta, M.R., Bennett, C.L., Halpern, M., Hinshaw, G., Jarosik, N., Kogut, A., Limon, M., Meyer, S.S., Page, L., Tucker, G.S., Weiland, J.L., Wollack, E., Wright, E.L.: Astrophys. J. Suppl. Ser. 148, 175 (2003)

Spergel, D.N., Bean, R., Dor e, O., Nolta, M.R., Bennett, C.L., Dunkley, J., Hinshaw, G., Jarosik, N., Komatsu, E., Page, L., et al.: Astrophys. J. Suppl. Ser. 170, 377 (2007)

Starobinsky, A.A.: Soviet Journal of Experimental and Theoretical Physics Letters 86, 157 (2007)

Tegmark, M., Strauss, M.A., Blanton, M.R., Abazajian, K., Dodelson, S., Sandvik, H., Wang, X., Weinberg, D.H., Zehavi, I., Bahcall, N.A., et al.: Phys. Rev. D 69(10), 103501 (2004)

Tsujikawa, S., Uddin, K., Mizuno, S., Tavakol, R., Yokoyama, J.: Phys. Rev. D 77(10), 103009 (2008)

Tsujikawa, S., Gannouji, R., Moraes, B., Polarski, D.: Phys. Rev. D 80(8), 084044 (2009)

Wang, L., Steinhardt, P.J.: Astrophys. J. 508, 483 (1998)

Weinberg, S.: Reviews of Modern Physics 61, 1 (1989) 


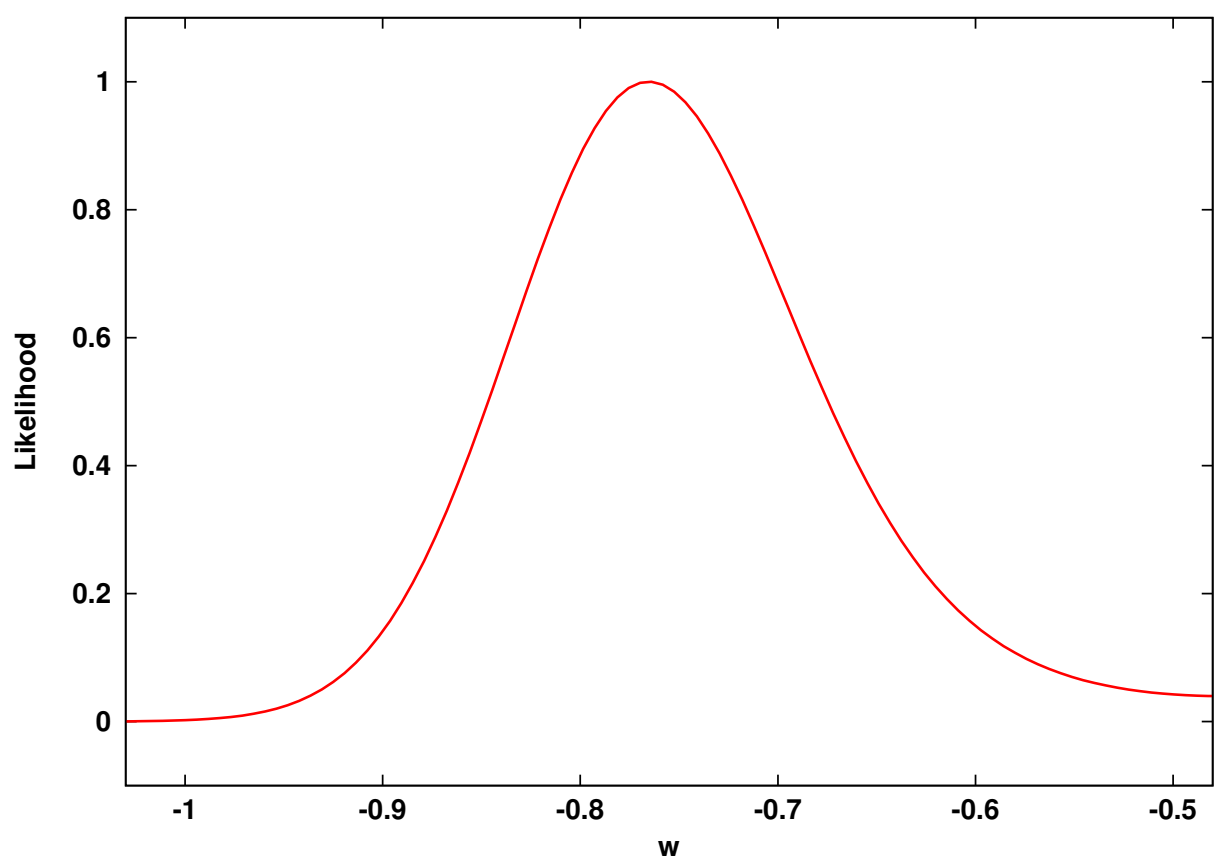

